\title{
Infinite-order scale-setting using the principle of maximum conformality: A remarkably efficient method for eliminating renormalization scale ambiguities for perturbative QCD
}

\author{
Leonardo Di Giustino $\odot,{ }^{1,2, *}$ Stanley J. Brodsky $\oplus^{2, \dagger}$ Sheng-Quan Wang, ${ }^{2,3, \ddagger}$ and Xing-Gang Wu ${ }^{4, \S}$ \\ ${ }^{1}$ Department of Science and High Technology, University of Insubria, \\ via Valleggio 11, I-22100 Como, Italy \\ ${ }^{2}$ SLAC National Accelerator Laboratory, Stanford University, Stanford, California 94039, USA \\ ${ }^{3}$ Department of Physics, Guizhou Minzu University, Guiyang 550025, People's Republic of China \\ ${ }^{4}$ Department of Physics, Chongqing University, Chongqing 401331, People's Republic of China
}

(Received 5 February 2020; accepted 23 June 2020; published 10 July 2020)

\begin{abstract}
We identify a property of renormalizable $\mathrm{SU}(\mathrm{N}) / \mathrm{U}(1)$ gauge theories, intrinsic conformality (iCF), which underlies the scale invariance of physical observables and leads to a remarkably efficient method to solve the conventional renormalization scale ambiguity at every order in perturbative QCD (pQCD): the PMC ${ }_{\infty}$. This new method reflects the underlying conformal properties displayed by pQCD at next-to-next-toleading order, eliminates the scheme dependence of pQCD predictions, and is consistent with the general properties of the principle of maximum conformality (PMC). We introduce a new method to identify conformal and $\beta$-terms which can be applied from either a numerical or an analytical calculations. We illustrate the $\mathrm{PMC}_{\infty}$ for the thrust and $\mathrm{C}$-parameter distributions in $e^{+} e^{-}$annihilation and then show how to apply this new method to general observables in QCD. We point out how the implementation of the $\mathrm{PMC}_{\infty}$ can significantly improve the precision of pQCD predictions; its implementation in a multiloop analysis also simplifies the calculation of higher order corrections in a general renormalizable gauge theory.
\end{abstract}

DOI: 10.1103/PhysRevD.102.014015

\section{INTRODUCTION}

A key issue in making precise predictions in QCD is the uncertainty in setting the renormalization scale $\mu_{R}$ in order to determine the correct running coupling $\alpha_{s}\left(\mu_{R}^{2}\right)$ in the perturbative expansion of a scale-invariant quantity. The conventional practice of simply guessing the scale $\mu_{R}$ of the order of a typical momentum transfer $Q$ in the process, and then varying the scale over a range $Q / 2$ and $2 Q$, gives predictions which depend on the renormalization scheme, retains dependence on the initial scale choice, leads to a nonconformal series which diverges as $\sim \alpha_{s}^{n} \beta_{0}^{n} n$ !, and is even invalid for QED [1]. In fact, a physical process will depend on many invariants and thus have multiple renormalization scales which depend on the dynamics of the process. Other proposals for renormalization scale settings such as PMS [2] or FAC [3] not only have the same

\footnotetext{
*leonardo.digiustino@gmail.com

†sjbth@slac.stanford.edu

sqwang@cqu.edu.cn

${ }^{\S}$ wuxg@cqu.edu.cn
}

Published by the American Physical Society under the terms of the Creative Commons Attribution 4.0 International license. Further distribution of this work must maintain attribution to the author(s) and the published article's title, journal citation, and DOI. Funded by SCOAP. difficulties, but they also lead to incorrect and unphysical results [4]. It has been shown recently how all the theoretical constraints can be satisfied at once, leading to accurate results by using the principle of maximum conformality (PMC) [5-7]. The primary purpose of the PMC method is to solve the scale-setting ambiguity; it has been extended to all orders $[8,9]$, and it determines the correct running coupling and the correct momentum flow, accordingly, to the RGE invariance $[10,11]$. This leads to results that are invariant with respect to the initial renormalization scale, in agreement with the requirement of scale invariance of an observable in pQCD [12].

We show here how the implementation at all orders of the PMC simplifies in many cases by identifying only the $\beta_{0^{-}}$ terms at each order of accuracy due to the presence of a new property of the perturbative corrections. First we recall that there is no ambiguity in setting the renormalization scale in QED. The standard Gell-Mann-Low scheme determines the correct renormalization scale by setting the scale to the virtuality of the exchanged photon [13]. For example, in electron-muon elastic scattering, the renormalization scale is the virtuality of the exchanged photon, i.e., the spacelike momentum transfer squared $\mu_{R}^{2}=q^{2}=t$. Thus,

$$
\alpha(t)=\frac{\alpha\left(t_{0}\right)}{1-\Pi\left(t, t_{0}\right)}
$$


where

$$
\Pi\left(t, t_{0}\right)=\frac{\Pi(t)-\Pi\left(t_{0}\right)}{1-\Pi\left(t_{0}\right)} .
$$

From Eq. (1) it follows that the renormalization scale $\mu_{R}=t$ can be determined by the $\beta_{0}$-term at the lowest order. This scale is sufficient to sum all the vacuum polarization contributions into the dressed photon propagator, both proper and improper at all orders. Again in QED, considering the case of two-photon exchange, a new, different scale is introduced in order to absorb all the $\beta$-terms related to the new subset or subprocess into the scale. Also in this case the scale can be determined by identifying the lowest order $\beta_{0^{-}}$ term alone. This term identifies the virtuality of the exchanged momenta causing the running of the scale in that subprocess. This scale again would sum all the contributions related to the $\beta$-function into the renormalization scale, and no further corrections need to be introduced to the scale at higher orders. Given that the $\mathrm{pQCD}$ and $\mathrm{pQED}$ predictions match analytically in the $N_{C} \rightarrow 0$ limit where $C_{F} \alpha_{\mathrm{QCD}} \rightarrow \alpha_{\mathrm{QED}}$ (see Ref. [14]), we extend the same procedure to $\mathrm{pQCD}$. In fact, in many cases in $\mathrm{QCD}$, the $\beta_{0}$ terms alone can determine the pQCD renormalization scale at all orders [15], eliminating the renormalon contributions $\alpha_{s}^{n} \beta_{0}^{n} n$ !. Though in non-Abelian theories other diagrams related to the three- and four-gluon vertices arise, these terms do not necessarily spoil this procedure. In fact, in $\mathrm{QCD}$, the $\beta_{0}$ terms arising from the renormalization of the three-gluon or four-gluon vertices as well as from gluon wave-function renormalization determine the renormalization scales of the respective diagrams, and no further corrections to the scales need to be introduced at higher orders. In conclusion, if we focus on a particular class of diagrams, we can fix the PMC scale by determining the $\beta_{0}$-term alone, and we show this to be connected to the general scale invariance of an observable in a gauge theory. In this article, we introduce a parametrization of the observables which stems directly from the analysis of the perturbative QCD corrections and which reveals interesting properties like scale invariance independently of the process or the kinematics. We point out that this parametrization can be an intrinsic general property of gauge theories, and we define this property as intrinsic conformality $\left(\mathrm{iCF}^{1}\right)$. We also show how this property directly indicates what the correct renormalization scale $\mu_{R}$ is at each order of calculation, and we define this new method as $\mathrm{PMC}_{\infty}$ : infinite-order scale-setting using the principle of maximum conformality. We discuss the iCF property and the $\mathrm{PMC}_{\infty}$ for the case of the thrust and C-parameter distributions in $e^{+} e^{-} \rightarrow$ 3 jets, and we show the results.

In general, a normalized IR-safe single-variable observable, such as the thrust distribution for the $e^{+} e^{-} \rightarrow 3$ jets $[16,17]$, is the sum of pQCD contributions calculated up to next-to-next-to-leading order (NNLO) at the initial renormalization scale $\mu_{0}$ :

\footnotetext{
${ }^{1}$ Here, the conformality is intended as RG invariance only.
}

$$
\begin{aligned}
\frac{1}{\sigma_{0}} \frac{O d \sigma\left(\mu_{0}\right)}{d O}= & \left\{\frac{\alpha_{s}\left(\mu_{0}\right)}{2 \pi} \frac{O d A_{O}\left(\mu_{0}\right)}{d O}+\left(\frac{\alpha_{s}\left(\mu_{0}\right)}{2 \pi}\right)^{2} \frac{O d B_{O}\left(\mu_{0}\right)}{d O}\right. \\
& \left.+\left(\frac{\alpha_{s}\left(\mu_{0}\right)}{2 \pi}\right)^{3} \frac{O d C_{O}\left(\mu_{0}\right)}{d O}+O\left(\alpha_{s}^{4}\right)\right\}
\end{aligned}
$$

where is the $\sigma_{0}$ is tree-level hadronic cross section; the $A_{O}$, $B_{O}$, and $C_{O}$ are, respectively, the LO, NLO, and NNLO coefficients; and $O$ is the selected nonintegrated variable. For the sake of simplicity, we will refer to the differential coefficients as to implicit coefficients, and we drop the derivative symbol, i.e.,

$$
\begin{aligned}
& A_{O}\left(\mu_{0}\right) \equiv \frac{O d A_{O}\left(\mu_{0}\right)}{d O}, \quad B_{O}\left(\mu_{0}\right) \equiv \frac{O d B_{O}\left(\mu_{0}\right)}{d O}, \\
& C_{O}\left(\mu_{0}\right) \equiv \frac{O d C_{O}\left(\mu_{0}\right)}{d O}
\end{aligned}
$$

We define here the intrinsic conformality as the property of a renormalizable $\mathrm{SU}(\mathrm{N}) / \mathrm{U}(1)$ gauge theory, like QCD, which yields to a particular structure of the perturbative corrections that can be made explicit by representing the perturbative coefficients using the following parametrization ${ }^{2}$ :

$$
\begin{aligned}
A_{O}\left(\mu_{0}\right)= & A_{\mathrm{Conf}}, \\
B_{O}\left(\mu_{0}\right)= & B_{\mathrm{Conf}}+\frac{1}{2} \beta_{0} \ln \left(\frac{\mu_{0}^{2}}{\mu_{I}^{2}}\right) A_{\mathrm{Conf}}, \\
C_{O}\left(\mu_{0}\right)= & C_{\mathrm{Conf}}+\beta_{0} \ln \left(\frac{\mu_{0}^{2}}{\mu_{\mathrm{II}}^{2}}\right) B_{\mathrm{Conf}} \\
& +\frac{1}{4}\left[\beta_{1}+\beta_{0}^{2} \ln \left(\frac{\mu_{0}^{2}}{\mu_{I}^{2}}\right)\right] \ln \left(\frac{\mu_{0}^{2}}{\mu_{I}^{2}}\right) A_{\mathrm{Conf}},
\end{aligned}
$$

where $A_{\text {Conf }}, B_{\text {Conf }}$, and $C_{\text {Conf }}$ are the scale-invariant conformal coefficients (i.e., the coefficients of each perturbative order not depending on the scale $\mu_{0}$ ) while we define $\mu_{N}$ as intrinsic conformal scales and $\beta_{0}, \beta_{1}$ are the first two coefficients of the $\beta$-function. We note that the implicit coefficients are defined at the scale $\mu_{0}$ and that they change according to the standard RG equations under a change of the renormalization scale according to

$$
\begin{aligned}
A_{O}\left(\mu_{R}\right)= & A_{O}\left(\mu_{0}\right), \\
B_{O}\left(\mu_{R}\right)= & B_{O}\left(\mu_{0}\right)+\frac{1}{2} \beta_{0} \ln \left(\frac{\mu_{R}^{2}}{\mu_{0}^{2}}\right) A_{O}\left(\mu_{0}\right), \\
C_{O}\left(\mu_{R}\right)= & C_{O}\left(\mu_{0}\right)+\beta_{0} \ln \left(\frac{\mu_{R}^{2}}{\mu_{0}^{2}}\right) B_{O}\left(\mu_{0}\right) \\
& +\frac{1}{4}\left[\beta_{1}+\beta_{0}^{2} \ln \left(\frac{\mu_{R}^{2}}{\mu_{0}^{2}}\right)\right] \ln \left(\frac{\mu_{R}^{2}}{\mu_{0}^{2}}\right) A_{O}\left(\mu_{0}\right) .
\end{aligned}
$$

\footnotetext{
${ }^{2}$ We neglect other running parameters here such as the mass terms.
} 
It can be shown that the form of Eq. (4) is scale invariant, and it is preserved under a change of the renormalization scale from $\mu_{0}$ to $\mu_{R}$ by standard RG equations (5), i.e.,

$$
\begin{aligned}
A_{O}\left(\mu_{R}\right)= & A_{\mathrm{Conf}}, \\
B_{O}\left(\mu_{R}\right)= & B_{\mathrm{Conf}}+\frac{1}{2} \beta_{0} \ln \left(\frac{\mu_{R}^{2}}{\mu_{I}^{2}}\right) A_{\mathrm{Conf}}, \\
C_{O}\left(\mu_{R}\right)= & C_{\mathrm{Conf}}+\beta_{0} \ln \left(\frac{\mu_{R}^{2}}{\mu_{\mathrm{II}}^{2}}\right) B_{\mathrm{Conf}} \\
& +\frac{1}{4}\left[\beta_{1}+\beta_{0}^{2} \ln \left(\frac{\mu_{R}^{2}}{\mu_{I}^{2}}\right)\right] \ln \left(\frac{\mu_{R}^{2}}{\mu_{I}^{2}}\right) A_{\mathrm{Conf}} .
\end{aligned}
$$

We notice that the form of Eq. (4) is invariant and that the initial scale dependence is exactly removed by $\mu_{R}$. Extending this parametrization to all orders, we achieve a scale-invariant quantity: The iCF parametrization is a sufficient condition in order to obtain a scale-invariant observable.

In order to show this property, we collect the terms identified by the same conformal coefficient, we call each set a conformal subset, and we extend the property to the order $n$ :

$$
\begin{aligned}
\sigma_{I}= & \left\{\left(\frac{\alpha_{s}\left(\mu_{0}\right)}{2 \pi}\right)+\frac{1}{2} \beta_{0} \ln \left(\frac{\mu_{0}^{2}}{\mu_{I}^{2}}\right)\left(\frac{\alpha_{s}\left(\mu_{0}\right)}{2 \pi}\right)^{2}\right. \\
+ & \left.\frac{1}{4}\left[\beta_{1}+\beta_{0}^{2} \ln \left(\frac{\mu_{0}^{2}}{\mu_{I}^{2}}\right)\right] \ln \left(\frac{\mu_{0}^{2}}{\mu_{I}^{2}}\right)\left(\frac{\alpha_{s}\left(\mu_{0}\right)}{2 \pi}\right)^{3}+\ldots\right\} A_{\mathrm{Conf}} \\
\sigma_{\mathrm{II}}= & \left\{\left(\frac{\alpha_{s}\left(\mu_{0}\right)}{2 \pi}\right)^{2}+\beta_{0} \ln \left(\frac{\mu_{0}^{2}}{\mu_{\mathrm{II}}^{2}}\right)\left(\frac{\alpha_{s}\left(\mu_{0}\right)}{2 \pi}\right)^{3}+\ldots\right\} B_{\mathrm{Conf}} \\
\sigma_{\mathrm{III}}= & \left\{\left(\frac{\alpha_{s}\left(\mu_{0}\right)}{2 \pi}\right)^{3}+\ldots\right\} C_{\mathrm{Conf}}, \\
& \vdots . \\
\sigma_{n}= & \left\{\left(\frac{\alpha_{s}\left(\mu_{0}\right)}{2 \pi}\right)^{n}\right\} \mathcal{L}_{n \text { Conf }} .
\end{aligned}
$$

In each subset we have only one intrinsic scale and only one conformal coefficient, and the subsets are disjoint; then no mixing terms among the scales or the coefficients are introduced in this parametrization. The structure of the subsets remains invariant under a global change of the renormalization scale as shown from Eq. (6). The structure of each conformal set $\sigma_{I}, \sigma_{\mathrm{II}}, \sigma_{\mathrm{III}}, \ldots$ and, consequently, the $\mathrm{iCF}$ are also preserved if we fix a different renormalization scale for each conformal subset, i.e.,

$$
\left(\mu^{2} \frac{\partial}{\partial \mu^{2}}+\beta\left(\alpha_{s}\right) \frac{\partial}{\partial \alpha_{s}}\right) \sigma_{n}=0
$$

We define here the property of Eq. (7) of separating an observable in the union of ordered scale-invariant disjoint subsets $\sigma_{I}, \sigma_{\mathrm{II}}, \sigma_{\mathrm{III}}, \ldots$ as ordered scale invariance.
In order to extend the $\mathrm{iCF}$ to all orders, we first define a partial limit $J_{/ n} \rightarrow \infty$ as the limit obtained by including the higher order corrections relative only to those $\beta_{0}, \beta_{1}, \beta_{2}, \ldots, \beta_{n-2}$ terms that have been determined already at order $n$ for each subset. Then we perform the complementary $\bar{n}$ limit which consists in including all the remaining terms. For the $J_{/ n}$ limit we have

$$
\begin{aligned}
\lim _{J_{n} \rightarrow \infty} \sigma_{\mathrm{I}} \rightarrow & \left(\frac{\left.\alpha_{s}\left(\mu_{\mathrm{I}}\right)\right|_{n-2}}{2 \pi}\right) A_{\mathrm{Conf}} \\
\lim _{J_{/ n} \rightarrow \infty} \sigma_{\mathrm{II}} \rightarrow & \left(\frac{\left.\alpha_{s}\left(\mu_{\mathrm{II}}\right)\right|_{n-3}}{2 \pi}\right)^{2} B_{\mathrm{Conf}} \\
\lim _{J_{/ n} \rightarrow \infty} \sigma_{\mathrm{III}} \rightarrow & \left(\frac{\left.\alpha_{s}\left(\mu_{\mathrm{III}}\right)\right|_{n-4}}{2 \pi}\right)^{3} C_{\mathrm{Conf}} \\
\vdots & \vdots \\
\lim _{J_{/ n} \rightarrow \infty} \sigma_{n} \equiv & \left(\frac{\alpha_{s}\left(\mu_{0}\right)}{2 \pi}\right)^{n} \mathcal{L}_{n \mathrm{Conf}}
\end{aligned}
$$

where $\left.\alpha_{s}\left(\mu_{I}\right)\right|_{n-2}$ is the coupling calculated up to $\beta_{n-2}$ at the intrinsic scale $\mu_{I}$. Given the particular ordering of the powers of the coupling, in each conformal subset we have the coefficients of the $\beta_{0}, \ldots, \beta_{n-k-1}$ terms, where $k$ is the order of the conformal subset and $n$ is the order of the highest subset with no $\beta$-terms. We notice that the limit of each conformal subset is finite and scale invariant up to $\sigma_{n-1}$. The remaining scale dependence is confined in the coupling of the $n$th term. Any combination of the $\sigma_{I}, \ldots, \sigma_{n-1}$ subsets is finite and scale invariant. We can now extend the iCF to all orders by performing the $\bar{n}$ limit. In this limit we include all the remaining higher order corrections. For the calculated conformal subsets this leads to defining the coupling at the same scales but including all the missing $\beta$ terms. Thus, each conformal subset remains scale invariant. We point out that we are not making any assumption on the convergence of the series for this limit. Then we have

$$
\begin{aligned}
& \lim _{\bar{n} \rightarrow \infty} \sigma_{\mathrm{I}} \rightarrow\left(\frac{\alpha_{s}\left(\mu_{\mathrm{I}}\right)}{2 \pi}\right) A_{\mathrm{Conf}} \\
& \lim _{\bar{n} \rightarrow \infty} \sigma_{\mathrm{II}} \rightarrow\left(\frac{\alpha_{s}\left(\mu_{\mathrm{II}}\right)}{2 \pi}\right)^{2} B_{\mathrm{Conf}} \\
& \lim _{\bar{n} \rightarrow \infty} \sigma_{\mathrm{III}} \rightarrow\left(\frac{\alpha_{s}\left(\mu_{\mathrm{III}}\right)}{2 \pi}\right)^{3} C_{\mathrm{Conf}} \\
& \vdots \quad \vdots \\
& \lim _{\bar{n} \rightarrow \infty} \sigma_{n} \equiv \lim _{n \rightarrow \infty}\left(\frac{\alpha_{s}\left(\mu_{0}\right)}{2 \pi}\right)^{n} \mathcal{L}_{n \mathrm{Conf}} \rightarrow \text { Conformal Limit }
\end{aligned}
$$

where $\alpha_{s}\left(\mu_{I}\right)$ is the complete coupling determined at the same scale $\mu_{I}$. Equation (10) shows that the whole renormalization scale dependence has been completely 
removed. In fact, neither the intrinsic scales $\mu_{N}$ nor the conformal coefficients $A_{\text {Conf }}, B_{\text {Conf }}, C_{\text {Conf }}, \ldots, \mathcal{L}_{n \text { Conf }}, \ldots$ depend on the particular choice of the initial scale. The only term with a residual $\mu_{0}$ dependence is the $n$-term, but this dependence cancels in the limit $n \rightarrow \infty$. The scale dependence is totally confined in the coupling $\alpha_{s}\left(\mu_{0}\right)$, and its behavior does not depend on the particular choice of any scale $\mu_{0}$ in the perturbative region, i.e., $\lim _{n \rightarrow \infty} \alpha_{s}\left(\mu_{0}\right)^{n} \sim a^{n}$ with $a<1$. Hence the limit of $\lim _{n \rightarrow \infty} \sigma_{n}$ depends only on the properties of the theory and not on the scale of the coupling in the perturbative regime. The proof given here shows that the $\mathrm{iCF}$ is sufficient to have a scale-invariant observable, and it does not depend on the particular convergence of the series. In order to show the necessary condition, we separate the two cases of a convergent series and an asymptotic expansion. For the first case the necessary condition stems directly from the uniqueness of the $\mathrm{iCF}$ form since, given a finite limit and the scale invariance, any other parametrization can be reduced to the $\mathrm{iCF}$ by means of appropriate transformations in agreement with the RG equations. For the second case, an asymptotic expansion, though not convergent, can be truncated at a certain order $n$, which is the case of Eq. (7). Given the particular structure of the iCF, we can perform the first partial limit $J_{/ n}$, and we would achieve a finite and scale-invariant prediction, $\sigma_{N-1}=\sum_{i=1}^{n-1} \sigma_{i}$, for a truncated asymptotic expansion, as shown in Eq. (9). Given the truncation of the series in the region of maximum convergence, the $n$th term would be reduced to the lowest value, so the scale dependence of the observable would reach its minimum. Given the finite and scale-invariant limit $\sigma_{N-1}$, we conclude that the iCF is unique and then necessary for an ordered scale-invariant truncated asymptotic expansion up to the $n$th order. We point out that, in general, the $\mathrm{iCF}$ form is the most general and irreducible parametrization which leads to the scale invariance. Other parametrizations are forbidden since if we introduce more scales $^{3}$ in the logarithms of one subset, we would spoil the invariance under the RG transformation, and we could not achieve Eq. (6); on the other hand, no scale dependence can be introduced in the intrinsic scales since it would remain in the observable already in the first partial limit $J_{/ n}$ and it could not be eliminated. The conformal coefficients are conformal quantities by definition at each order; thus, they do not depend on the renormalization scale, and they do not have a perturbative expansion. Hence, the $\mathrm{iCF}$ is a necessary and sufficient condition for scale invariance.

\section{ICF AND ORDERED SCALE INVARIANCE}

The iCF parametrization can stem from either an inner property of the theory, the $\mathrm{iCF}$, or from direct

\footnotetext{
${ }^{3}$ Here we refer to the form of Eq. (4). In principle, it is possible to write other parametrizations preserving the scale invariance, but these can be reduced to the iCF by means of appropriate transformations in agreement with the RG equations.
}

parametrization of the scale-invariant observable. In both cases the iCF parametrization makes the scale dependence of the observable explicit, and it exactly preserves the scale invariance. Once we have defined an observable in the iCF form, we not only have the scale invariance of the entire observable but also the ordered scale invariance (i.e., the scale invariance of each subset $\sigma_{n}$ or $\sigma_{N-1}$ ). The latter property is crucial in order to obtain scale-invariant observables independently from the particular kinematical region and independently from the starting order of the observable or the order of the truncation of the series. Since, in general, a theory is blind with respect to the particular observable/ process that we investigate, the theory should preserve the ordered scale invariance in order to always define scaleinvariant observables. Hence, if the iCF is an inner property of the theory, it leads to implicit coefficients that are neither independent nor conformal. This is made explicit in Eq. (4), but it is hidden in the perturbative calculations in the case of the implicit coefficients. For instance, the presence of the iCF clearly reveals when a particular kinematical region is approached and the $A_{O}$ becomes null. This would cause a break of the scale invariance since a residual initial scale dependence would remain in the observable in the higher order coefficients. The presence of the iCF solves this issue by leading to the correct redefinition of all the coefficients at each order, preserving the correct scale invariance exactly. Thus, in the case of a scale-invariant observable $O$ defined, according to the implicit form [Eq. (2)], by the coefficients $\left\{A_{O}, B_{O}, C_{O}, \ldots, O_{O}, \ldots\right\}$, it cannot simply undergo the change $\rightarrow\left\{0, B_{O}, C_{O}, \ldots, O_{O}, \ldots\right\}$ since this would break the scale invariance. In order to preserve the scale invariance, we must redefine the coefficients $\left\{\tilde{A}_{O}=\right.$ $\left.0, \tilde{B}_{O}, \tilde{C}_{O}, \ldots, \tilde{O}_{O}, \ldots\right\}$ canceling out all the initial scale dependence originating from the $\mathrm{LO}$ coefficient $A_{O}$ at all orders. This is equivalent to subtracting out a whole invariant conformal subset $\sigma_{I}$ related to the coefficient $A_{\text {Conf }}$ from the scale-invariant observable $O$. This mechanism is clear in the case of the explicit form of the iCF, Eq. (4), where if $A_{\text {Conf }}=0$ then the whole conformal subset is null and the scale invariance is preserved. We underline that the conformal coefficients can acquire all the possible values without breaking the scale invariance; they contain the essential information on the physics of the process, while all the correlation factors can be reabsorbed in the renormalization scales as shown by the PMC method [5-9]. Hence, if a theory has the property of the ordered scale invariance, it exactly preserves the scale invariance of observables independently from the process, the kinematics, and the starting order of the observable. We suggest that if a theory has intrinsic conformality, all the renormalized quantities, such as cross sections, can be parametrized with the iCF form. This property should be preserved by the renormalization scheme or by the definition of IR-safe quantities, and it should also be preserved in observables defined in effective theories. 


\section{THE PMC $_{\infty}$}

We introduce here a new method to eliminate the scalesetting ambiguity in single-variable scale-invariant distributions called $\mathrm{PMC}_{\infty}$. This method is based on the original PMC principle [5-9] and agrees with all the different formulations for the PMC scales at the lowest order. Essentially, the core of the $\mathrm{PMC}_{\infty}$ is the same as all the BLM-PMC prescriptions [18]; i.e., the correct running coupling value, and hence its renormalization scale at the lowest order, is identified by the $\beta_{0}$-term at each order or, equivalently, by the intrinsic conformal scale $\mu_{N}$. The $\mathrm{PMC}_{\infty}$ preserves the $\mathrm{iCF}$, and then the scale invariance by absorbing an infinite set of $\beta$-terms at all orders. This method differs from the other PMC prescriptions since, due to the presence of the intrinsic conformality, no perturbative correction in $\alpha_{s}$ needs to be introduced at higher orders in the PMC scales. Given that all the $\beta$-terms of a single conformal subset are included in the renormalization scale already with the definition at lowest order, no initial scale or scheme dependence is left due to the unknown $\beta$-terms in each subset. The $\mathrm{PMC}_{\infty}$ scale of each subset can be unambiguously determined by the $\beta_{0}$-term of each order, we underline that all logarithms of each subset have the same argument, and all the differences arising at higher orders have to be included only in the conformal coefficients. Reabsorbing all the $\beta$-terms into the scale, the $\beta_{0}^{n} n$ ! terms (related to the renormalons [19]) are also eliminated; thus, the precision is improved, and the perturbative QCD predictions can be extended to a wider range of values. The initial scale dependence is totally confined in the unknown $\mathrm{PMC}_{\infty}$ scale of the last order of accuracy [i.e., up to the NNLO case in $\alpha_{s}\left(\mu_{0}\right)^{3}$ ]. Thus, if we fix the renormalization scale independently to the proper intrinsic scale for each subset $\mu_{N}$, we end up with a perturbative sum of totally conformal contributions up to the order of accuracy:

$$
\begin{aligned}
& \frac{1}{\sigma_{0}} \frac{O d \sigma\left(\mu_{\mathrm{I}}, \mu_{\mathrm{II}}, \mu_{\mathrm{III}}\right)}{d O} \\
& =\left\{\frac{\alpha_{s}\left(\mu_{\mathrm{I}}\right)}{2 \pi} \frac{O d A_{\mathrm{Conf}}}{d O}+\left(\frac{\alpha_{s}\left(\mu_{\mathrm{II}}\right)}{2 \pi}\right)^{2} \frac{O d B_{\mathrm{Conf}}}{d O}\right. \\
& \left.\quad+\left(\frac{\alpha_{s}\left(\mu_{\mathrm{III}}\right)}{2 \pi}\right)^{3} \frac{O d C_{\mathrm{Conf}}}{d O}\right\}+\mathcal{O}\left(\alpha_{s}^{4}\right),
\end{aligned}
$$

where, at this order, $\mu_{\mathrm{III}}=\mu_{0}$.

\section{ICF COEFFICIENTS AND SCALES}

We describe here how all the coefficients of Eq. (4) can be identified from either a numerical or an analytical perturbative calculation. We will use as a template the NNLO thrust distribution results calculated in Refs. [20,21]. Since the leading order is already $\left(A_{\text {Conf }}\right)$ void of $\beta$-terms, we start with NLO coefficients. A general numerical or theoretical calculation keeps track of all the color factors and the respective coefficients:

$$
B_{O}\left(N_{f}\right)=C_{F}\left[C_{A} B_{O}^{N_{c}}+C_{F} B_{O}^{C_{F}}+T_{F} N_{f} B_{O}^{N_{f}}\right]
$$

where $C_{F}=\frac{\left(N_{c}^{2}-1\right)}{2 N_{c}}, C_{A}=N_{c}$ and $T_{F}=1 / 2$. The dependence on $N_{f}$ is made explicit here for the sake of clarity. We can determine the conformal coefficient $B_{\text {Conf }}$ of the NLO order straightforwardly; by fixing the number of flavors $N_{f}$ in order to kill the $\beta_{0}$ term,

$$
\begin{aligned}
B_{\mathrm{Conf}} & =B_{O}\left(N_{f} \equiv \frac{33}{2}\right), \\
B_{\beta_{0}} & \equiv \log \frac{\mu_{0}^{2}}{\mu_{I}^{2}}=2 \frac{B_{O}-B_{\mathrm{Conf}}}{\beta_{0} A_{\mathrm{Conf}}},
\end{aligned}
$$

we would achieve the same results in the usual PMC way, i.e., identifying the $N_{f}$ coefficient with the $\beta_{0}$ term and then determining the conformal coefficient. Both methods are consistent, and results for the intrinsic scales and the coefficients are in perfect agreement. At the NNLO a general coefficient is made from the contribution of six different color factors:

$$
\begin{aligned}
C_{O}\left(N_{f}\right)= & \frac{C_{F}}{4}\left\{N_{c}^{2} C_{O}^{N_{c}^{2}}+C_{O}^{N_{c}^{0}}+\frac{1}{N_{c}^{2}} C_{O}^{\frac{1}{N_{c}^{2}}}+N_{f} N_{c} \cdot C_{O}^{N_{f} N_{c}}\right. \\
& \left.+\frac{N_{f}}{N_{c}} C_{O}^{N_{f} / N_{c}}+N_{f}^{2} C_{O}^{N_{f}^{2}}\right\} .
\end{aligned}
$$

In order to identify all the terms of Eq. (4), we notice first that the coefficients of the terms $\beta_{0}^{2}$ and $\beta_{1}$ are already given by the NLO coefficient $B_{\beta_{0}}$; then, we need to determine only the $\beta_{0}$ terms and the conformal $C_{\mathrm{Conf}}$-terms. In order to determine the latter coefficients, we use the same procedure we used for the NLO; i.e., we set the number of flavors $N_{f} \equiv 33 / 2$ in order to drop all the $\beta_{0}$ terms. We then have

$$
\begin{aligned}
C_{\mathrm{Conf}}= & C_{O}\left(N_{f} \equiv \frac{33}{2}\right)-\frac{1}{4} \bar{\beta}_{1} B_{\beta_{0}} A_{\mathrm{Conf}}, \\
C_{\beta_{0}} \equiv & \log \left(\frac{\mu_{0}^{2}}{\mu_{\mathrm{II}}^{2}}\right)=\frac{1}{\beta_{0} B_{\mathrm{Conf}}}\left(C_{O}-C_{\mathrm{Conf}}\right. \\
& \left.-\frac{1}{4} \beta_{0}^{2} B_{\beta_{0}}^{2} A_{\mathrm{Conf}}-\frac{1}{4} \beta_{1} B_{\beta_{0}} A_{\mathrm{Conf}}\right),
\end{aligned}
$$

with $\bar{\beta}_{1} \equiv \beta_{1}\left(N_{f}=33 / 2\right)=-107$. This procedure can be extended at every order, and one may decide whether to cancel $\beta_{0}, \beta_{1}$, or $\beta_{2}$ by fixing the appropriate number of flavors. The results can be compared, leading to an exact determination of all the coefficients. We point out that by extending the intrinsic conformality to all orders, we can predict, at this stage, the coefficients of all the color factors of the higher orders related to the $\beta$-terms, except those related to the higher order conformal coefficients and $\beta_{0}$-terms (e.g., at NNNLO, the $D_{\text {Conf }}$ and $D_{\beta_{0}}$ ). The $\beta$-terms are coefficients that stem from UV-divergent diagrams connected with the running of the coupling constant and not from UV-finite diagrams. UV-finite $N_{F}$ terms may arise but would not 
contribute to the $\beta$-terms. On the contrary, $N_{f}$ terms coming from UV-divergent diagrams, depending dynamically on the virtuality of the underlying quark and gluon subprocesses, have to be considered as $\beta$-terms, and they would determine the intrinsic conformal scales. In general, each $\mu_{N}$ is an independent function of $\sqrt{s}$ and of the selected variable $O$, and it varies with the number of colors $N_{c}$ mainly due to $g g g-$ and $g g g g$-vertices. The latter terms arise at higher orders only in non-Abelian theory, but they do not spoil the iCF form. We underline that the $\mathrm{iCF}$ applies to scale-invariant singlevariable distributions; in case one is interested in the renormalization of a particular diagram, e.g., the $g g g$-vertex, contributions from different $\beta$-terms should be singled out in order to identify the respective intrinsic conformal scale consistently with the renormalization of the non-Abelian ggg-vertex, as shown in [22].

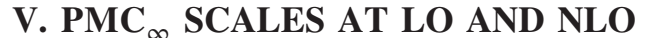

According to the $\mathrm{PMC}_{\infty}$ prescription, we fix the renormalization scale to $\mu_{N}$ at each order, absorbing all the $\beta$ terms into the coupling. We notice a small mismatch between the zeroes of the conformal coefficient $B_{\text {Conf }}$ and those of the numerator in Eq. (15). Due to our limited knowledge of the strong coupling at low energies, in order to avoid singularities in the NLO scale $\mu_{\mathrm{II}}$, we introduce a regularization which leads to a finite scale $\tilde{\mu}_{\mathrm{II}}$. These singularities stem from a rather logarithmic behavior of the conformal coefficients when low values of the variable $1-T$ are approached. Large logarithms arise from the IR divergence cancellation procedure, and they can be resummed in order to restore a predictive perturbative regime [23-28]. We point out that IR cancellation should not spoil the iCF property, and an IR cancellation Monte Carlo technique consistent with the iCF would be required. Whether this is an actual deviation from the iCF form has to be further investigated. However, since the discrepancies between the coefficients are rather small, we introduce a regularization method based on redefinition of the norm of the coefficient $B_{\text {Conf }}$ in order to cancel out these singularities in the $\mu_{\mathrm{II}}$ scale. This regularization is consistent with the PMC principle, and up to the accuracy of the calculation, it does not introduce any bias effect in the results or ambiguity in the NLO-PMC ${ }_{\infty}$ scale. All the differences introduced by the regularization would start at the NNNLO of accuracy, and they can be absorbed later in the higher order $\mathrm{PMC}_{\infty}$ scales. Thus, the first two $\mathrm{PMC}_{\infty}$ scales result in

$$
\begin{aligned}
& \mu_{\mathrm{I}}=\sqrt{s} \cdot e^{-\frac{1}{2} B_{\beta_{0}},} \quad(1-T)<0.33,
\end{aligned}
$$

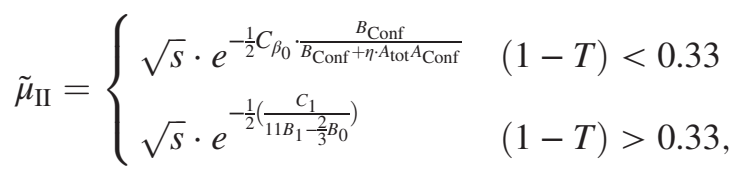

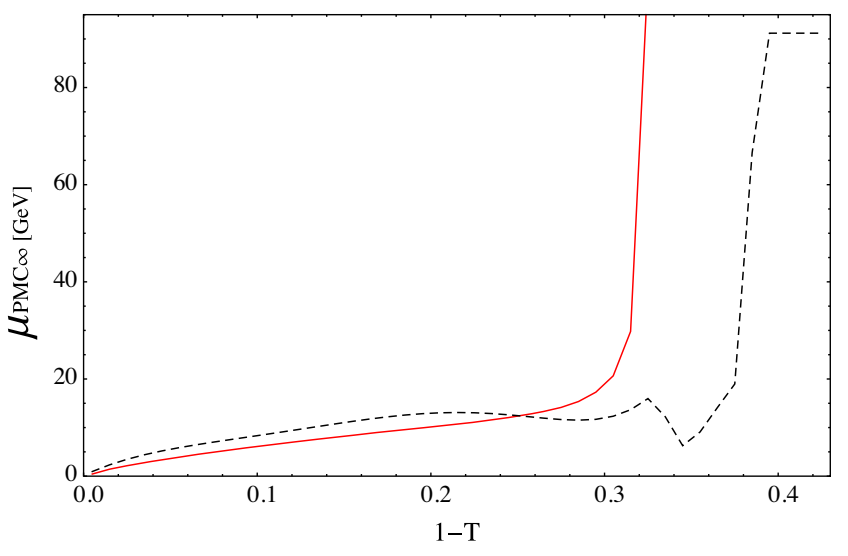

FIG. 1. The LO-PMC 1 $_{\infty}$ (solid red) and the NLO-PMC ${ }_{\infty}$ (dashed black) scales for thrust.

where $\sqrt{s}=M_{Z_{0}}$, and the value of $\eta=3.51$ has been fixed by matching the zeroes of the numerator and the denominator of $C_{\beta_{0}}$. We have to point out that in the region $(1-T)>0.33$, we have a clear example of $\mathrm{iCF}$, where the kinematical constraints set $A_{\mathrm{Conf}}=0$. According to Eq. (6), by setting $A_{\text {Conf }}=0$ the whole conformal subset $\sigma_{I}$ becomes null. In this case all the $\beta$ terms at NLO and NNLO disappear except the $\beta_{0}$-term at NNLO which determines the $\mu_{\text {II }}$ scale. The surviving $N_{f}$ terms at NLO or the $N_{f}^{2}$ at NNLO are related to the finite $N_{F}$-term at NLO and to the mixed $N_{f} \cdot N_{F}$ term arising from $B_{O} \cdot \beta_{0}$ at NNLO. Using the following parametrization,

$$
\begin{aligned}
& A_{O}=0, \\
& B_{O}=B_{0}+B_{1} \cdot N_{f}, \\
& C_{O}=C_{0}+C_{1} \cdot N_{f}+C_{2} \cdot N_{f}^{2},
\end{aligned}
$$

we can determine $\tilde{\mu}_{\mathrm{II}}$ for the region $(1-T)>0.33$ as shown in Eq. (17),

$$
\tilde{\mu}_{\mathrm{II}}=\sqrt{s} e^{-\frac{1}{2}\left(\frac{C_{1}}{11 B_{1}-\frac{2}{3} B_{0}}\right)},
$$

by identifying the $\beta_{0}$-term at NNLO. The LO and NLO $\mathrm{PMC}_{\infty}$ scales are shown in Fig. 1. We notice that the two $\mathrm{PMC}_{\infty}$ scales have similar behaviors in the range $(1-T)<0.33$ and the $\mathrm{LO}-\mathrm{PMC}_{\infty}$ scale agrees with the PMC scale used in [29]. This method totally eliminates both the ambiguity in the choice of the renormalization scale and the scheme dependence at all orders in QCD.

\section{NNLO THRUST DISTRIBUTION RESULTS}

Here we use the results of Refs. [20,21], and for the running coupling $\alpha_{s}(Q)$ we use the RunDec program [30]. In order to consistently normalize the thrust distribution, we expand the denominator in $\alpha_{0} \equiv \alpha_{s}\left(\mu_{0}\right)$, while the couplings in the numerator are renormalized at different $\mathrm{PMC}_{\infty}$ 
scales: $\alpha_{I} \equiv \alpha_{s}\left(\mu_{I}\right), \alpha_{\mathrm{II}} \equiv \alpha_{s}\left(\tilde{\mu}_{\mathrm{II}}\right)$. We point out here that the proper normalization would be given by the integration of the total cross section after renormalization with the $\mathrm{PMC}_{\infty}$ scales; nonetheless, since the $\mathrm{PMC}_{\infty}$ prescription involves only absorption of higher order terms into the scales, the difference would be within the accuracy of the calculations, i.e., $\sim \mathcal{O}\left(\alpha_{s}^{4}\left(\mu_{0}\right)\right)$. The experimentally measured thrust distribution is normalized to the total hadronic cross section $\sigma_{\text {tot }}$ as follows:

$\frac{1}{\sigma_{\mathrm{tot}}} \frac{O d \sigma\left(\mu_{I}, \mu_{\mathrm{II}}, \mu_{0}\right)}{d O}=\left\{\bar{\sigma}_{I}+\bar{\sigma}_{\mathrm{II}}+\bar{\sigma}_{\mathrm{III}}+\mathcal{O}\left(\alpha_{s}^{4}\right)\right\}$

where

$$
\sigma_{\mathrm{tot}}=\sigma_{0}\left(1+\frac{\alpha_{s}\left(\mu_{0}\right)}{2 \pi} A_{\mathrm{tot}}+\left(\frac{\alpha_{s}\left(\mu_{0}\right)}{2 \pi}\right)^{2} B_{\mathrm{tot}}+O\left(\alpha_{s}^{3}\right)\right)
$$

is the total integrated cross section and $A_{\mathrm{tot}}, B_{\mathrm{tot}}$ are

$$
\begin{gathered}
A_{\mathrm{tot}}=\frac{3}{2} C_{F}, \\
B_{\mathrm{tot}}=\frac{C_{F}}{4} N_{c}+\frac{3}{4} C_{F} \frac{\beta_{0}}{2}(11-8 \zeta(3))-\frac{3}{8} C_{F}^{2} .
\end{gathered}
$$

The normalized subsets in the region $(1-T)<0.33$ are then

$$
\begin{aligned}
\bar{\sigma}_{I}= & A_{\mathrm{Conf}} \frac{\alpha_{I}}{2 \pi}, \\
\bar{\sigma}_{\mathrm{II}}= & \left(B_{\mathrm{Conf}}+\eta A_{\mathrm{tot}} A_{\mathrm{Conf}}\right)\left(\frac{\alpha_{\mathrm{II}}}{2 \pi}\right)^{2}-\eta A_{\mathrm{tot}} A_{\mathrm{Conf}}\left(\frac{\alpha_{0}}{2 \pi}\right)^{2} \\
& -A_{\mathrm{tot}} A_{\mathrm{Conf}} \frac{\alpha_{0}}{2 \pi} \frac{\alpha_{I}}{2 \pi}, \\
\bar{\sigma}_{\mathrm{III}}= & \left(C_{\mathrm{Conf}}-A_{\mathrm{tot}} B_{\mathrm{Conf}}-\left(B_{\mathrm{tot}}-A_{\mathrm{tot}}^{2}\right) A_{\mathrm{Conf}}\right)\left(\frac{\alpha_{0}}{2 \pi}\right)^{3} .
\end{aligned}
$$

Normalized subsets for the region $(1-T)>0.33$ can be achieved simply by setting $A_{\text {Conf }} \equiv 0$ in Eq. (23). Within the numerical precision of these calculations, there is no evidence of the presence of spurious terms, such as UVfinite $N_{f}$ terms up to NNLO [31]. These terms must be rather small or comparable with numerical fluctuations. In addition, we notice a small, rather constant difference between the iCF-predicted and the calculated coefficient for the $N_{f}^{2}$-color factor of Ref. [20], which might be due to an $N_{f}^{2}$ UV-finite coefficient or possibly to statistics. This small difference must be included in the conformal coefficient, and it has a complete negligible impact on the total thrust distribution. In Fig. 2 we show the thrust distribution at NLO and at NNLO using the $\mathrm{PMC}_{\infty}$ method. Theoretical errors for the thrust distribution at NLO and at NNLO are

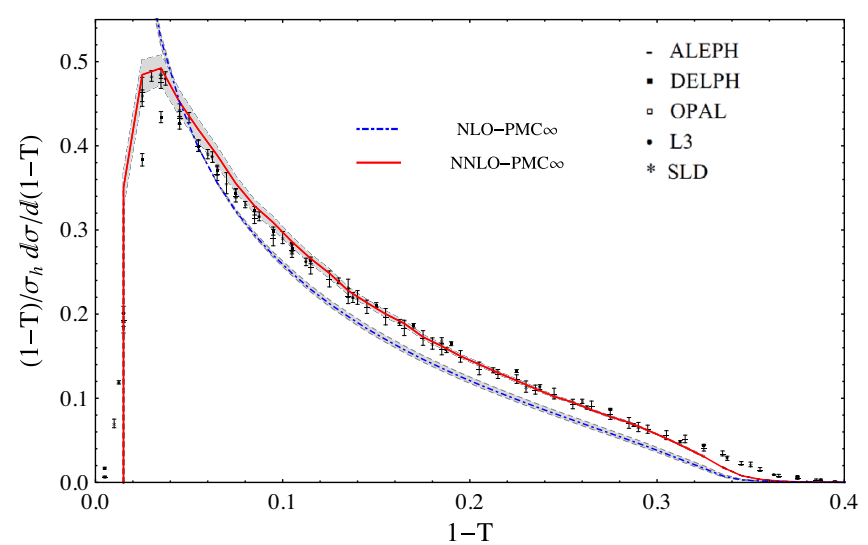

FIG. 2. The thrust distribution under the $\mathrm{PMC}_{\infty}$ at NLO (dotdashed blue) and at NNLO (solid red). The experimental data points are taken from the ALEPH, DELPHI, OPAL, L3, and SLD experiments [33-37]. The shaded area shows theoretical errors for the $\mathrm{PMC}_{\infty}$ predictions at NLO and at NNLO.

also shown (the shaded area). Conformal quantities are not affected by a change of renormalization scale. Thus, the errors shown give an evaluation of the level of conformality achieved up to the order of accuracy, and they have been calculated using standard criteria, i.e., varying the remaining initial scale value in the range $\sqrt{s} / 2 \leq \mu_{0} \leq 2 \sqrt{s}$. Using the same definition of the parameter $\bar{\delta}$ as in Ref. [32], we have in the interval $0 .<(1-T)<0.33$ average errors of $\bar{\delta} \simeq 3.54 \%$ and $1.77 \%$ for the thrust at NLO and at NNLO, respectively. A larger improvement has been calculated in the range $0 .<(1-T)<0.42$ from $\bar{\delta} \simeq$ $7.36 \%$ to $1.95 \%$ from NLO to the NNLO accuracy with the $\mathrm{PMC}_{\infty}$.

In Fig. 3 a direct comparison of the $\mathrm{PMC}_{\infty}$ with the conventional scale setting results (obtained in [20] and

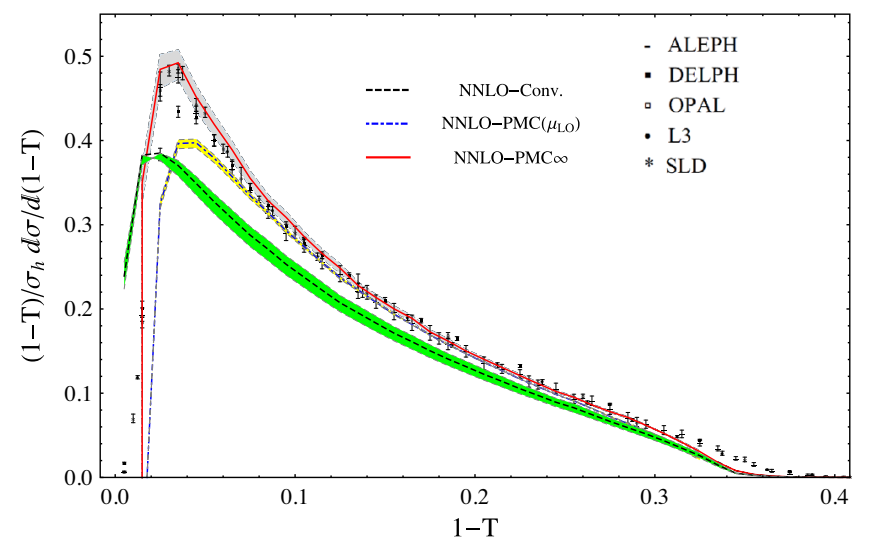

FIG. 3. The thrust distribution at NNLO under the conventional (dashed black), the $\mathrm{PMC}\left(\mu_{\mathrm{LO}}\right)$ (dot-dashed blue), and the $\mathrm{PMC}_{\infty}$ (solid red). The experimental data points are taken from the ALEPH, DELPHI, OPAL, L3, and SLD experiments [33-37]. The shaded areas show theoretical error predictions at NNLO, and they have been calculated by varying the remaining initial scale value in the range $\sqrt{s} / 2 \leq \mu_{0} \leq 2 \sqrt{s}$. 
TABLE I. Average error $\bar{\delta}$, for NNLO thrust distribution under conventional, $\mathrm{PMC}\left(\mu_{\mathrm{LO}}\right)$, and $\mathrm{PMC}_{\infty}$ scale settings calculated in different ranges of values of the $(1-T)$ variable.

\begin{tabular}{cccc}
\hline \hline $\bar{\delta}[\%]$ & Conventional & $\mathrm{PMC}\left(\mu_{\mathrm{LO}}\right)$ & $\mathrm{PMC}_{\infty}$ \\
\hline $0.10<(1-T)<0.33$ & 6.03 & 1.41 & 1.31 \\
$0.21<(1-T)<0.33$ & 6.97 & 2.19 & 0.98 \\
$0.33<(1-T)<0.42$ & 8.46 & & 2.61 \\
$0.00<(1-T)<0.33$ & 5.34 & 1.33 & 1.77 \\
$0.00<(1-T)<0.42$ & 6.00 & & 1.95 \\
\hline \hline
\end{tabular}

$[32,38])$ is shown. In addition, we have also shown the results of the first PMC approach used in [29], which we indicate as $\operatorname{PMC}\left(\mu_{\mathrm{LO}}\right)$ extended to the NNLO accuracy. In this approach the last unknown PMC scale $\mu_{\mathrm{NLO}}$ of the NLO has been set to the last known PMC scale $\mu_{\mathrm{LO}}$ of the LO, while the NNLO scale $\mu_{\mathrm{NNLO}} \equiv \mu_{0}$ has been left unset and varied in the range $\sqrt{s} / 2 \leq \mu_{0} \leq 2 \sqrt{s}$. This analysis has been performed in order to show that the procedure of setting the last unknown scale to the last known one leads to stable and precise results and is consistent with the proper PMC method in a wide range of values of the $(1-T)$ variable.

Average errors calculated in different regions of the spectrum are reported in Table I. From the comparison with the conventional scale setting, we notice that the $\mathrm{PMC}_{\infty}$ prescription significantly improves the theoretical predictions. In addition, results are in remarkable agreement with the experimental data in a wider range of values $(0.015 \leq 1-T \leq 0.33)$, and they show an improvement of the $\operatorname{PMC}\left(\mu_{L O}\right)$ results when the two-jet and multijet regions are approached, i.e., the region of the peak and the region $(1-T)>0.33$, respectively. The use of the $\mathrm{PMC}_{\infty}$ approach on perturbative thrust QCD calculations restores the correct behavior of the thrust distribution in the region $(1-T)>0.33$, and this is a clear effect of the iCF property. Comparison with the experimental data has been improved all over the spectrum, and the introduction of the $N^{3} L O$ correction would improve this comparison, especially in the multijet $1-T>0.33$ region. In the $\mathrm{PMC}_{\infty}$ method theoretical errors are given by the unknown intrinsic conformal scale of the last order of accuracy. We expect that this scale will not be significantly different from that of the previous orders. In this particular case, as shown in Eq. (23), we also have a dependence on the initial scale $\alpha_{s}\left(\mu_{0}\right)$ left due to the normalization and to the regularization terms. These errors represent $12.5 \%$ and $1.5 \%$, respectively, of all of the theoretical errors in the range $0<(1-T)<0.42$, and they could be improved by means of a correct normalization.

\section{NNLO C-PARAMETER DISTRIBUTION RESULTS}

The same analysis applies straightforwardly to the C-parameter distribution, including the regularizing $\eta$

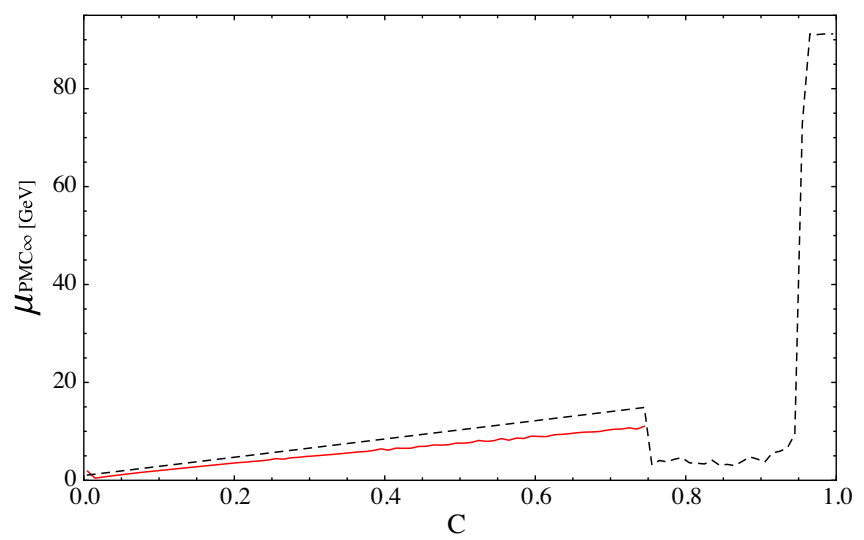

FIG. 4. The LO-PMC (solid red) and the NLO-PMC $_{\infty}$ (dashed black) scales for the C-parameter.

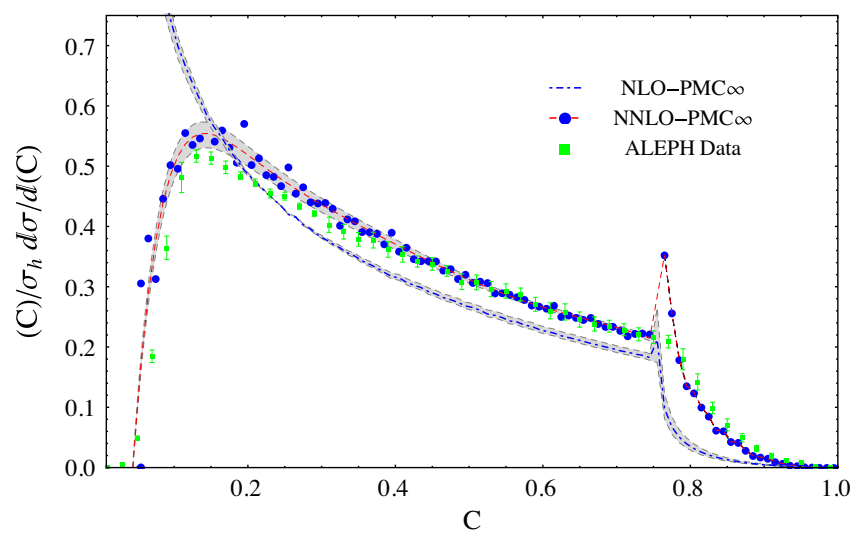

FIG. 5. The C-parameter distribution under the $\mathrm{PMC}_{\infty}$ at NLO (dot-dashed blue) and at NNLO (dashed red). Blue points indicate the NNLO-PMC ${ }_{\infty}$ thrust distribution obtained with $\mu_{\mathrm{III}}=\mu_{0}=M_{Z_{0}}$. The experimental data points (green) are taken from the ALEPH experiment [33]. Dashed lines of the NNLO distribution show fits of the theoretical calculations with interpolating functions for the values of the remaining initial scale $\mu_{0}=2 M_{Z_{0}}$ and $M_{Z_{0}} / 2$. The shaded area shows theoretical errors for the $\mathrm{PMC}_{\infty}$ predictions at NLO and at NNLO calculated by varying the remaining initial scale value in the range $\sqrt{s} / 2 \leq \mu_{0} \leq 2 \sqrt{s}$.

parameter which has been set to the same value of 3.51 . The same scales of Eqs. (16) and (17) apply to the C-parameter distribution in the region $0<C<0.75$ and in the region $0.75<C<1$. In fact, due to kinematical

TABLE II. Average error $\bar{\delta}$ for NNLO C-parameter distribution under conventional, $\mathrm{PMC}\left(\mu_{\mathrm{LO}}\right)$, and $\mathrm{PMC}_{\infty}$ scale settings calculated in different ranges of values of the $(C)$ variable.

\begin{tabular}{cccc}
\hline \hline $\bar{\delta}[\%]$ & Conventional & $\mathrm{PMC}\left(\mu_{\mathrm{LO}}\right)$ & $\mathrm{PMC}_{\infty}$ \\
\hline $0.00<(C)<0.75$ & 4.77 & 0.85 & 2.43 \\
$0.75<(C)<1.00$ & 11.51 & 3.68 & 2.42 \\
$0.00<(C)<1.00$ & 6.47 & 1.55 & 2.43 \\
\hline \hline
\end{tabular}




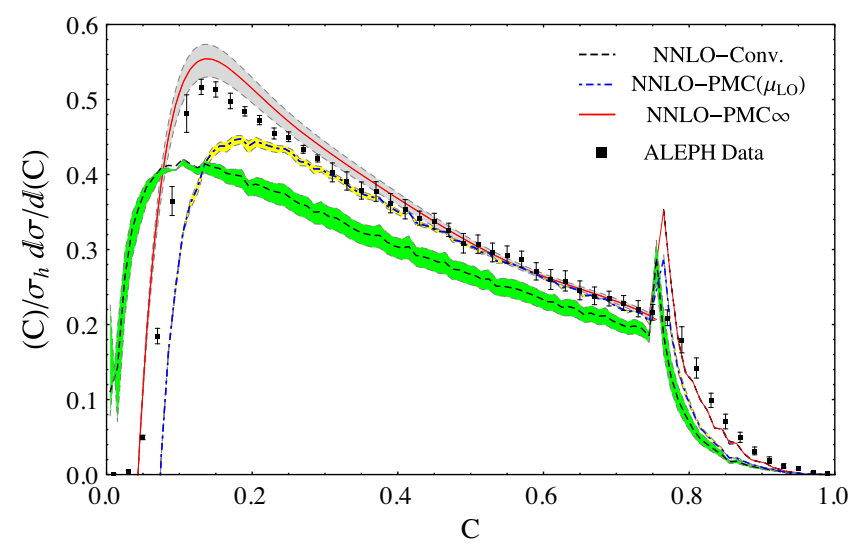

FIG. 6. The NNLO C-parameter distribution under the conventional scale setting (dashed black), the $\operatorname{PMC}\left(\mu_{\mathrm{LO}}\right)$ (dot-dashed blue) and the $\mathrm{PMC}_{\infty}$ (solid red). The experimental data points (black) are taken from the ALEPH experiment [33]. The shaded area shows theoretical error predictions at NNLO calculated by varying the remaining initial scale value in the range $\sqrt{s} / 2 \leq \mu_{0} \leq 2 \sqrt{s}$.

constraints that set $A_{\text {Conf }}=0$, we also have the same iCF effect for the C-parameter. Results for the C-parameter scales and distributions are shown in Figs. 4 and 5, respectively.

Theoretical errors have been calculated, as in the previous case, using standard criteria, and results indicate an average error over the whole spectrum $0<(C)<1$ of the C-parameter distribution at NLO and at NNLO of $\bar{\delta} \simeq$ $7.26 \%$ and $2.43 \%$, respectively.

A comparison of average errors according to the different methods is shown in Table II. Results show that the $\mathrm{PMC}_{\infty}$ improves the NNLO QCD predictions for the C-parameter distribution over the whole spectrum.

A comparison of the distributions calculated with the conventional scale setting, the $\operatorname{PMC}\left(\mu_{\mathrm{LO}}\right)$ [39] and the $\mathrm{PMC}_{\infty}$ is shown in Fig. 6. Results for the $\mathrm{PMC}_{\infty}$ show a remarkable agreement with the experimental data away from the regions $C<0.05$ and $C \simeq 0.75$. The errors due to the normalization and to the regularization terms [Eq. (23)] are, respectively, $8.8 \%$ and $0.7 \%$ of all of the theoretical errors. The perturbative calculations could be further improved using a correct normalization and also by introducing the large logarithms resummation technique in order to extend the perturbative regime.

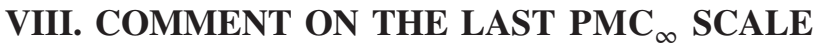

In this article we have shown a property of the perturbative QCD corrections which is consistent with the MC results of single-variable distributions for $e^{+} e^{-} \rightarrow 3$ jets. This property leads to the $\mathrm{PMC}_{\infty}$ : an infinite-order scale setting based on the principle of maximum conformality method. The $\mathrm{PMC}_{\infty}$ method preserves the iCF form and is void of ambiguities. The absence of ambiguities in $\mathrm{PMC}_{\infty}$ is clearly shown here by simply noticing that the new scale at each order is fixed by considering the $\beta_{0}$-term, i.e., $N_{f^{-}}$ term, and no other term is needed, in agreement with the $\mathrm{iCF}$ parametrization. Thus, the $\mathrm{PMC}_{\infty}$ scale fixing procedure is constrained by the iCF form, and the choice of the scales is totally free of any ambiguity. The only unknown scale which remains unfixed, apparently, is the one given by the last order of accuracy. In this article this scale has been fixed to $\mu_{\mathrm{III}}=\mu_{0}=M_{Z_{0}}$ for the sake of a consistent comparison with the conventional method. We remark that the last "unknown" PMC scale can be fixed to the last known one. In fact, as we have also shown in this article, in general, the differences between two consecutive scales are rather small, and the use of the method $\operatorname{PMC}\left(\mu_{\mathrm{LO}}\right)$ leads to precise and consistent results in a wide range of the perturbative region, as reported in Tables I and II of the previous sections. In the case of the thrust and C-parameter distributions calculated at NNLO with the $\mathrm{PMC}_{\infty}$, the differences between the $\mu_{\mathrm{III}}=M_{Z_{0}}$ and $\mu_{\mathrm{III}}=\tilde{\mu}_{\mathrm{II}}$ are also negligible, and these differences are totally confined within the errors shown for the distributions in the perturbative region. Given the conformal limit of Eq. (10), the last term in the iCF determines the level of conformality reached by the expansion, and the entire scale dependence is confined in the coupling. As previously shown, this term is the main source of the uncertainties (as also reported in Ref. [40]), and a further investigation regarding the regularization method applied here would be necessary in order to extend the procedure to all orders.

\section{CONCLUSIONS}

We have introduced here the $\mathrm{iCF}$, a parametrization which exactly preserves the scale invariance of an observable. We have shown a new method to solve the conventional renormalization scale ambiguity in QCD, called $\mathrm{PMC}_{\infty}$, which preserves the iCF and leads to conformal renormalization scales. This method agrees with the PMC at LO, and it applies to observables, especially in cases of a clearly manifested iCF, for example, in the case of the event shape variables distributions. We have presented a new procedure to identify the $\mathrm{iCF} / \mathrm{PMC}_{\infty}$ coefficients at all orders, which can be applied to either numerical or analytical calculations. The $\mathrm{PMC}_{\infty}$ has been applied to the NNLO thrust and C-parameter distributions, and the results show perfect agreement with the experimental data. The evaluation of theoretical errors using standard criteria shows that the $\mathrm{PMC}_{\infty}$ significantly improves the theoretical predictions in all of the spectra of the shape variables. The $\mathrm{PMC}_{\infty}$ method eliminates the renormalon growth $\alpha_{s}^{n} \beta_{0}^{n} n$ !, the scheme dependence, and all the uncertainties related to the scale ambiguity up to the order of accuracy. The $\mathrm{iCF} / \mathrm{PMC}_{\infty}$ scales are identified by the lowest order logarithm related to the $\beta_{0}$-term at each order, and all the physics of the process is contained in the conformal 
coefficients. This is in complete agreement with QED and with the Gell-Mann and Low scheme [13,14]. We have discussed why iCF should be considered as a strict requirement for a theory in order to preserve the scale invariance of the observables, and we have shown that iCF is consistent with the single-variable thrust and C-parameter distributions. We point out that other conformal aspects of QCD resulting from different sectors such as commensurate scale relations (CSR) [41] or dual theories such as AdS/CFT [42] might also be related to the intrinsic conformality. We emphasize that the iCF property in a theory would have two main remarkable consequences: First, it shows what the correct coupling constant is at each order as a function of the conformal intrinsic scale $\mu_{N}$, and second, since only the conformal and the $\beta_{0}$ coefficients need to be identified in the observables at each order, by means of the $\mathrm{PMC}_{\infty}$ method, the iCF would reveal its predictive feature for the coefficients of the higher order color factors. We point out that in many cases the implementation of the $\mathrm{iCF}$ in a multiloop calculation procedure would lead to a significant reduction of the color factor coefficients, and it would speed up the calculations for higher order corrections.

\section{ACKNOWLEDGMENTS}

We thank Francesco Sannino for useful discussions. L. D. G. thanks the SLAC Theory Group for its kind hospitality and support. The work of S. J. B. was supported in part by the Department of Energy under Contract No. DE-AC0276SF00515. SLAC-PUB-17511.
[1] D. Maitre et al., Proc. Sci., PS-HEP2009 (2009) 367 [arXiv:0909.4949].

[2] P. M. Stevenson, Phys. Rev. D 23, 2916 (1981).

[3] G. Grunberg, Phys. Lett. 95B, 70 (1980).

[4] G. Kramer and B. Lampe, Z. Phys. A 339, 189 (1991).

[5] S. J. Brodsky and L. Di Giustino, Phys. Rev. D 86, 085026 (2012).

[6] S. J. Brodsky and X. G. Wu, Phys. Rev. D 85, 034038 (2012).

[7] S. J. Brodsky and X. G. Wu, Phys. Rev. Lett. 109, 042002 (2012).

[8] M. Mojaza, S. J. Brodsky, and X. G. Wu, Phys. Rev. Lett. 110, 192001 (2013).

[9] S. J. Brodsky, M. Mojaza, and X. G. Wu, Phys. Rev. D 89, 014027 (2014).

[10] X. G. Wu, J. M. Shen, B. L. Du, X. D. Huang, S. Q. Wang, and S. J. Brodsky, Prog. Part. Nucl. Phys. 108, 103706 (2019).

[11] X. G. Wu, Y. Ma, S. Q. Wang, H. B. Fu, H. H. Ma, S. J. Brodsky, and M. Mojaza, Rep. Prog. Phys. 78, 126201 (2015).

[12] X. G. Wu, S. J. Brodsky, and M. Mojaza, Prog. Part. Nucl. Phys. 72, 44 (2013).

[13] M. Gell-Mann and F.E. Low, Phys. Rev. 95, 1300 (1954).

[14] S. J. Brodsky and P. Huet, Phys. Lett. B 417, 145 (1998).

[15] S. J. Brodsky, J. R. Ellis, E. Gardi, M. Karliner, and M. A. Samuel, Phys. Rev. D 56, 6980 (1997).

[16] V. Del Duca, C. Duhr, A. Kardos, G. Somogyi, Z. Ször, Z. Tröcsanyi, and Z. Tulipánt, Phys. Rev. D 94, 074019 (2016).

[17] V. Del Duca, C. Duhr, A. Kardos, G. Somogyi, and Z. Tröcsanyi, Phys. Rev. Lett. 117, 152004 (2016).

[18] S. J. Brodsky, G. P. Lepage, and P. B. Mackenzie, Phys. Rev. D 28, 228 (1983).

[19] M. Beneke, Phys. Rep. 317, 1 (1999).
[20] S. Weinzierl, J. High Energy Phys. 06 (2009) 041.

[21] S. Weinzierl, Phys. Rev. Lett. 101, 162001 (2008).

[22] M. Binger and S. J. Brodsky, Phys. Rev. D 74, 054016 (2006).

[23] S. Catani, G. Turnock, B. R. Webber, and L. Trentadue, Phys. Lett. B 263, 491 (1991).

[24] S. Catani, L. Trentadue, G. Turnock, and B. R. Webber, Nucl. Phys. B407, 3 (1993).

[25] S. Catani, M. L. Mangano, P. Nason, and L. Trentadue, Nucl. Phys. B478, 273 (1996).

[26] U. Aglietti, L. Di Giustino, G. Ferrera, and L. Trentadue, Phys. Lett. B 651, 275 (2007).

[27] A. Banfi, H. McAslan, P. F. Monni, and G. Zanderighi, J. High Energy Phys. 05 (2015) 102.

[28] R. Abbate, M. Fickinger, A. H. Hoang, V. Mateu, and I. W. Stewart, Phys. Rev. D 83, 074021 (2011).

[29] S. Q. Wang, S. J. Brodsky, X. G. Wu, and L. Di Giustino, Phys. Rev. D 99, 114020 (2019).

[30] K. G. Chetyrkin, J. H. Kuhn, and M. Steinhauser, Comput. Phys. Commun. 133, 43 (2000).

[31] T. Gehrmann, N. Hfliger, and P. F. Monni, Eur. Phys. J. C 74, 2896 (2014).

[32] A. Gehrmann-De Ridder, T. Gehrmann, E. W. N. Glover, and G. Heinrich, Phys. Rev. Lett. 99, 132002 (2007).

[33] A. Heister et al. (ALEPH Collaboration), Eur. Phys. J. C 35, 457 (2004).

[34] J. Abdallah et al. (DELPHI Collaboration), Eur. Phys. J. C 29, 285 (2003).

[35] G. Abbiendi et al. (OPAL Collaboration), Eur. Phys. J. C 40, 287 (2005).

[36] P. Achard et al. (L3 Collaboration), Phys. Rep. 399, 71 (2004).

[37] K. Abe et al. (SLD Collaboration), Phys. Rev. D 51, 962 (1995).

[38] A. Gehrmann-De Ridder, T. Gehrmann, E. W. N. Glover, and G. Heinrich, J. High Energy Phys. 12 (2007) 094. 
[39] S. Q. Wang, S. J. Brodsky, X. G. Wu, J. M. Shen, and L. Di Giustino, Phys. Rev. D 100, 094010 (2019).

[40] H. A. Chawdhry and A. Mitov, Phys. Rev. D 100, 074013 (2019).
[41] H. J. Lu and S. J. Brodsky, Nucl. Phys. B, Proc. Suppl. 39BC, 309 (1995).

[42] S. J. Brodsky and G. F. de Tramond, Phys. Lett. B 582, 211 (2004). 